These results show conclusively that no important interference with the nitric-acid-ferrous-iron reaction is interposed by the organic matter present, and the method appears sufficiently promising to permit its use in the general estimation of aliphatic nitrate esters by those laboratories which do not have a nitrometer conveniently at hand.

It will be noted that the method gave low results with glycerine trinitrate, while with the mannite hexanitrate the results correspond closely with the calculated results. Glycerine trinitrate has an appreciable vapor tension ${ }^{7}$ at $100^{\circ}$ and part of this loss, at least, may have been due to the vaporization of the ester in steam before it was completely hydrolyzed. If $s 0$, this error could probably be eliminated by introducing the sample into a cold reaction mixture, followed by the gradual elevation of the reaction temperature. To insure absence of oxidation it would of course be necessary to replace the air by an inert gas.

'Titration of the residual ferrous iron with potassium permanganate is impossible when the mixture contains nitro compounds which develop interfering colors, notably trinitrotoluene. Though not investigated, this difficulty may apparently be diminished or possibly completely overcome by estimating the ferric iron through reduction by hydrogen iodide in acid solution, since the color change, from starch blue to the acid ferrous iron, should not be seriously affected by the yellow-to-red shades imparted by the interfering nitro compound.

\title{
Summary
}

Aliphatic nitrate esters may be estimated in the presence of certain nitro aromatic compounds by reduction with ferrous sulfate in excess, followed by titration of the unoxidized ferrous salt. A procedure sufficiently promising to permit its general use is described, together with suggestions for further experiments.

WASHINGTON, D. C.

[Contribution from the Chemical Iaboratory of the University of IILINOIS]

\section{THE PREPARATION OF 1,4-DIHALOGEN DERIVATIVES OF BUTANE}

\author{
By C. S. Marvel and A. L. Tanenbaum
}

Received August 17, 1922

The 1,4-dihalogen derivatives of butane are reagents that would be used more frequently in the organic laboratory if they were available. Several methods for their preparation have appeared in the literature but all are difficult to carry out. Probably the best method heretofore developed is that of $\mathrm{J}$. von Braun for the preparation of tetramethylene bromide by splitting benzoyl pyrrolidine with phosphorus pentabromide. ${ }^{1}$ This

"Marshall, "Explosives," P. Blakiston's Sons and Co., 1917, p. 735.

1 von Braun, Ber., 39, 4119-25 (1906). 
method, however, involves 7 reactions, some of which do not give satisfactory yields.

A series of reactions has been developed so that tetramethylene bromide can now be obtained much more readily than by the older methods. Several steps are similar to those used by von Braun but these have been somewhat improved and some of the more difficult steps have been avoided.

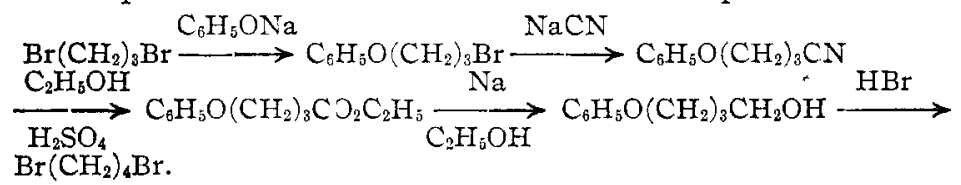

In this series of reactions the yields are good in almost every step. The first 3 reactions give yields of 80 to $95 \%$ of the theoretical amount. The reduction of the ester to the alcohol gives a yield of 62 to $68 \%$. The last reaction gives only a 40 to $45 \%$ yield of tetramethylene bromide but there is also formed phenoxybutyl bromide in 40 to $45 \%$ yields. This latter compound is a useful reagent, but if only tetramethylene bromide is desired, the phenoxybutyl bromide may be split down by further treatment with hydrobromic acid. The splitting of phenoxybutyl alcohol with hydriodic acid has also been studied, but the yields of the corresponding iodides are no better than those of the bromides obtained with hydrobromic acid.

The most interesting reaction of this series is the reduction of ethyl phenoxybutyrate to phenoxybutyl alcohol. The best method for the reduction of esters is that devised by Levene and Allen. ${ }^{2}$ In applying this method several facts have been learned that are very useful in carrying out the reaction. It is not necessary to use the special apparatus which has been described by the original workers, as very satisfactory results may be obtained by using a 3 -neck flask fitted with a reflux condenser, dropping funnel and mechanical stirrer. The main precaution that must be observed if good yields are to be obtained, is the use of alcohol that is free from water. If the ordinary absolute alcohol dried over quicklime is used in the reduction, the yield of phenoxybutyl alcohol is 40 to $45 \%$. When, however, this grade of alcohol is treated with a small amount of sodium and then distilled under conditions that exclude moisture, a very high grade of absolute alcohol is obtained. Using this latter grade of alcohol insures a 62 to $68 \%$ yield.

The alcohols which were prepared by Levene and Allen could be isolated by distillation of the reaction mixture with steam. With the higherboiling alcohols this method is not practical. The best method of isolating these latter alcohols is to remove the ethyl alcohol and toluene from a steam-bath and then, after treating the residue with water, to extract the high-boiling alcohol with ether.

2 I.evene and Allen, J. Biol. Chem, 27, (a) 435, (b) 443 (1916); (c) 33, 509 (1918). 
This method of reduction has also been applied to the esters of caprylic, lauric and myristic acids, and the corresponding alcohols have been obtained in yields of 65 to $75 \%$. Cyanides are also easily reduced to primary amines in the same way; thus, phenoxypropyl cyanide gives a yield of 80 to $87 \%$ of phenoxybutyl amine, which is much better than can be obtained by the ordinary method of carrying out a sodium alcohol reduction.

\section{Experimental Part}

Phenoxypropyl Bromide.-A mixture of 2 liters of water, $1 \mathrm{~kg}$. of trimethylene bromide and $370 \mathrm{~g}$. of phenol is heated to boiling under a reflux condenser and stirred with a mechanical stirrer. To the boiling solution is added over a period of 1 hour a solution of $150 \mathrm{~g}$. of sodium hydroxide in $500 \mathrm{cc}$. of water.

The mixture is stirred and heated for 4 to 5 hours longer in order to complete the reaction. The water layer is separated and discarded. The lower layer consisting of unchanged trimethylene bromide, phenoxypropyl bromide and diphenoxypropane is distilled under reduced pressure. The material boiling below $136^{\circ}$ at $20 \mathrm{~mm}$. is almost pure trimethylene bromide and weighs 340 to $380 \mathrm{~g}$. The fraction boiling at $136-142^{\circ}$ at $20 \mathrm{~mm}$. is pure phenoxypropyl bromide and weighs 560 to $600 \mathrm{~g}$. (an $84-85 \%$ yield, based on the trimethylene bromide actually used). On cooling to a low temperature the product crystallizes and melts at $7-8^{\circ}$. The high-boiling residue left in the distilling flask is diphenoxypropane ( 50 to $75 \mathrm{~g}$.).

The reaction has previously been carried out in alcoholic solution. ${ }^{3}$ The large excess of trimethylene bromide is quite necessary in order to avoid the formation of large quantitites of diphenoxypropane.

Phenoxypropyl Cyanide. -This preparation is carried out in essentially the same manner that has been described in the literature. ${ }^{4} \mathrm{~A}$ mixture of $1 \mathrm{~kg}$. of phenoxypropyl bromide, $325 \mathrm{~g}$. of sodium cyanide, $325 \mathrm{cc}$. of water and $500 \mathrm{~g}$. of $95 \%$ alcohol is refluxed on a steam cone for 15 to 20 hours. The solution is then cooled and treated with $800 \mathrm{cc}$. of benzene. The water layer is separated and extracted once with a little benzene $(100 \mathrm{cc}$.). The combined benzene extracts are distilled from a steam-bath until the solvent is removed. The residue is distilled under reduced pressure. The yield is 675 to $725 \mathrm{~g}$. (90-96\%) of phenoxypropyl cyanide boiling at $162-166^{\circ}$ at $22 \mathrm{~mm}$. As it cools the product crystallizes to a white solid melting at $41-42^{\circ}$. Melting points of $42^{\circ}$ and $46^{\circ}$ are given in the literature. ${ }^{5}$

Ethyl Phenoxybutyrate.-The preparation of this ester by the action of alcoholic sulfuric acid on phenoxypropyl cyanide has been mentioned in the literature ${ }^{b}$ but no details are given. A mixture of $625 \mathrm{~g}$. of $95 \%$ ethyl alcohol, $300 \mathrm{~g}$. of conc. sulfuric acid (sp. gr. 1.84) and $500 \mathrm{~g}$. of phenoxypropyl cyanide is refluxed for about 7 hours. The flask is cooled and ammonium sulfate separates. The liquid portion is drained into a separatory funnel and the lower portion is drawn off and added to the ammonium sulfate. The upper layer is nearly pure ester. Water is added to dissolve the ammonitum sulfate and the aqueous solution is extracted with a little benzene. The benzene extract is added to the main portion of the ester and this solution is washed with a solution of sodium carbonate to remove any acids that may be present. The ester is then distilled under reduced pressure. The yield is 495 to $516 \mathrm{~g}$. (75-80\%) of a product boiling at $160-165^{\circ}$ at $25 \mathrm{~mm}$.

s Ber., (a) 24, 2632 (1891); (b) 26, 2987 (1893); (c) Ref. 1, p. 4120.

"Ibid., (a) 24, 2640 (1891); (b) Ref. 1, p. 4121.

${ }^{5}$ Ref. 4a; ibid., 42, 2047 (1909).

- THIS JournaL, 42, 652 (1920). 
Phenoxybutyl Alcohol. - This compound has been prepared by the action of sodium nitrite on phenoxybutyl amine oxalate, ' but no physical constants have been recorded. It is easily obtained by the reduc ion of ethyl phenoxybutyrate with sodium and absolute $(100 \%)$ alcohol in toluene according to the general method of Levene and Allen. ${ }^{2 b}$

A 3-neck 3-liter round-bottom flask is fitted with a mechanical stirrer, dropping funnel and a reflux condenser. The condenser should be 2 meters long and should have an inner tube $2.5 \mathrm{~cm}$. in diameter. It is best connected to one of the necks of the reaction flask by means of a heavy piece of rubber tubing. The upper end of the condenser is wrotected with a calcium chloride tube. The stirrer should reach nearly to the bott on of the flask so that the stirring is very efficient.

In the flask are placed $110 \mathrm{~g}$. of sodium and $250 \mathrm{cc}$. of toluene previously dried over sodium. The flask is heated in an oil-bath until the sodium is melted and the stirrer is started. When the sodium is broken up into very small particles and well emulsified in the toluene, the oil-bath is removed and the flask is allowed to cool until the sodium has solidified. Vigorous stirring must be maintained to prevent the formation of large lumps. A solution of $100 \mathrm{~g}$. of ethyl phenoxybutyrate in $150 \mathrm{cc}$. of absolute (100\%) alconol is run in through the dropping funnel as rapidly as the condenser can take care of the vapors that are produced. This addition usually requires 3 to 4 minutes. Five hundred cc. of absolute $(100 \%)$ alcohol is then added as rapidly as possible, usually during 12 to 15 minutes. The reaction is allowed to proceed for about 15 minutes after the last of the alcollol has been added until all of the sodium is used up. Then about 3 in) ce. of water is added to decompose the sodium ethylate. The oil-bath is again arljusted to the flask and the solution is boiled for about 30 minutes to insure the hydrol$y$ sis of any unreduced ester. The flask is then fitted with a distilling head and the ethy alcohol-toluene mixture is distilled from the steam-bath until the alcohol is completely removed. The material remaining in the flask is treated with $500 \mathrm{cc}$. of water. The lower water layer is separated and extracted once with ether. The upper layer is treated with $200 \mathrm{cc}$. of water and $200 \mathrm{cc}$. of ether. The ether layer is separated and the water layer again extracted with ether. The comhined ether extract is washed with $100 \mathrm{cc}$. of water. The ether and any tolucne and water are then distilled under ordinary pressure and the phenoxybutyl alcohol is distilled under reduced pressure. The yield is 50 to $54 \mathrm{~g} .(62-68 \%)$ of a product boiling at $162-164^{\circ}$ at 19 mun.; $n_{\mathrm{D}}^{27}, 1.520$. Occasiona 1 yields of $75 \%$ have been obtained but the usual yields are somewhat less. As stated in the general discussion, the ethyl alcohol used in the reduction must be free from water. Density determinations on the dry distillate used in these experiments showed the product to be $100 \%$ ethyl alcohol. The apparatus must of course be dry in order to obtain good results.

An ice-bath should be available for cooling during the reduction as the reaction may become too violent if too much alcohol is added at once. If the reaction does become violent it may be checked by stopping the stirrer and cooling the flask in the ice-bath.

The phenoxybutyl alcohol was analyzed by the Parr total carbon method.

Analy'sis. Subs., $0.4470 ; 731.0$ ce. of $\mathrm{CO}_{2}\left(32.5^{\circ}, 739.5 \mathrm{~mm}\right.$. $)$. Calc. for $\mathrm{C}_{10} \mathrm{H}_{14} \mathrm{O}_{2}$ : C, 72.24. Found: 72.22.

As a characteristic solid derivative the $p$-nitrobenzoic acid ester was prepared by treating a little phenoxybutyl alcohol with $p$-nitrobenzoyl chloride. The ester after 2 crystallizations from alcohol melted sharply at $91^{\circ}$.

Analyses. Subs., 0.1368: $\mathrm{CO}_{2}, 0.3224 ; \mathrm{H}_{2} \mathrm{O}, 0.0652$. Calc. for $\mathrm{C}_{17} \mathrm{H}_{17} \mathrm{O}_{5} \mathrm{~N}: \mathrm{C}$, 64.73; H, 5.43. Found: C, 64.27; H, 5.34.

$N$-Octyl Alcohol.--This compound is easily obtained by reducing $75 \mathrm{~g}$. of ethyl caprylate with $85 \mathrm{~g}$. of sodium, $200 \mathrm{cc}$. of toluene and $550 \mathrm{cc}$ of absolute alcohol in the

i Ber, 42, 548 (1909). 
manner described before. The yield is 37 to $41 \mathrm{~g} .(67-75 \%)$ of octyl alcohol boiling at $103-105^{\circ}$ at $16 \mathrm{~mm}$.

Lauryl Alcohol.- Lauric ester was reduced by Levene and Allen ${ }^{8}$, but no details are given. They say the yield is less than $60 \%$. By reducing $100 \mathrm{~g}$. of ethyl laurate with $85 \mathrm{~g}$. of sodium, $200 \mathrm{cc}$. of toluene and $600 \mathrm{cc}$. of absolute alcohol, there is obtained ij:; to $57 \mathrm{~g}$. $(65-70 \%)$ of lauryl alcohol boiling at $143-146^{\circ}$ at $18 \mathrm{~mm}$. In working up the reaction mixture care must be taken to avoid emulsions when the water layer containing sodium hydroxide and sodium laurate (soap) is extracted with ether. If the residue after distilling the alcohol-toluene mixture is treated with $500 \mathrm{cc}$. of hot water and the aqueous layer separated, most of the soapy sodium laurate is removed in this solution and the remaining material may be worked up as usual.

Myristyl Alcohol.-This compound is obtained in 53 to $63 \mathrm{~g}$. yields $(63-75 \%)$ when $100 \mathrm{~g}$. of ethyl myristate is reduced with $85 \mathrm{~g}$. of sodium, $200 \mathrm{cc}$. of toluene and $600 \mathrm{cc}$. of absolute alcohol. The myristyl alcohol boils at $170-173^{\circ}$ under $20 \mathrm{~mm}$. pressure and melts at $39-39.5^{\circ}$. This agrees with the melting point recorded in the literature. ${ }^{9}$

Phenoxybutyl Amine.-The compound has been prepared by the reduction of thenoxypropyl cyanide with sodium and alcohol in the ordinary way. ${ }^{10}$ By reducing $100 \mathrm{~g}$. of phenoxy propyl cyanide with $100 \mathrm{~g}$. of sodium, $250 \mathrm{cc}$. of toluene and $650 \mathrm{cc}$. of atsolute alcohol, and purifying the reduction product by dissolving in hydrochloric acid and reprecipitating with alkali, there is obtained 82 to $90 \mathrm{~g}$. $(80-87 \%)$ of phenoxybityl amine boiling at $146-148^{\circ}$ under $17 \mathrm{~mm}$. pressure.

The Action of Hydrobromic Acid on Phenoxybutyl Alcohol.--Phenoxybutyl alcohol is converted into a mixture of phenoxybutyl bromide and tetramethylene bromide when it is heated with hydrobromic acid containing a little sulfuric acid. A mixture of $36 \mathrm{~g}$. of phenoxybutyl alcohol with $115 \mathrm{cc}$. of hydrobromic acid (sp. gr. 1.48) and $25 \mathrm{ce}$. of sulfuric acid (sp. gr. 1.84) is gently warmed for about an hour and then refluxed vigorously for about 8 hours. The reaction mixture is then cooled and diluted with $100 \mathrm{cc}$. of water. A mixture of tetramethylene bromide and phenoxybutyl bromide separates at the bottom and is drawn off. The water layer is washed once with benzene. The benzene extract is added to the main portion of the mixed bromides and the solution is extracted with $10 \%$ sodim hydroxide solution to remove the phenol. The benzene solution is dried over calcium chloride and distilled under reduced pressure. The solvent comes off first, then tetramethylene bromide boiling at $81-85^{\circ}$ at $18 \mathrm{~mm}$. and finally phenoxybutyl bromide boiling at $153-156^{\circ}$ at $18 \mathrm{~mm}$. The intermediate fractions are nesligible if a short column is used and care is taken to avoid superheating during the distillation. The yield is 18 to $20 \mathrm{~g}$. $(40-43 \%)$ of tetramethylene bromide and 19 to $20 \mathrm{~g}$. $(39-40 \%)$ of phenoxybutyl bromide.

The physical properties of the tetramethylene bromide thus obtained agree with those recorded in the literature. Phenoxybutyl bromide has not been previously deseribed. As it cools, it solidifies to a white solid. After crystallizing from alcohol, it melts sharply at $41^{\circ}$.

Analysis. Subs, 0.5211: 26.44 cc. of $0.0811 N$ AgNO . Calc. for $\mathrm{C}_{10} \mathrm{H}_{18} \mathrm{OBr}$ : Eit, 34.89. Found: 34.54 .

The yield of tetramethylene bromide cannot be greatly increased by heating the reaction mixture for a longer time. The yield is lowered if the sulfuric acid is not present. When fuming hydrobromic acid (sp. gr. 1.57) is used, the yield of tetramethylene bromide is increased to $45-47 \%$.

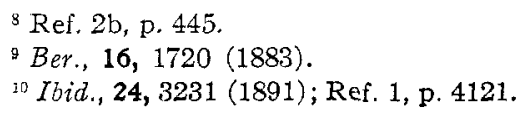


The reaction was carried out in sealed tubes at $150^{\circ}$ without increasing the yield. When higher temperatures were tried excessive decomposition took place.

The Action of Hydrobromic Acid on Phenoxybutyl Bromide.-By refluxing 46 g. of phenoxybutyl bromide with $1 \mathrm{CO} \mathrm{cc}$. of hydrobromic acid (sp. gr. 1.48) for 9 hours and working up the reaction mixture as described before, there is obtained $12 \mathrm{~g}$. (20\%) of tetramethylene bromide. Thirty-two g. of phenoxybutyl bromide is recovered.

Phenoxybutyl Bromide.- If only phenoxybutyl bromide is desired it may be obtained best by treating phenoxybutyl alcohol with phosphorous tribromide. Thirtythree $\mathrm{g}$. of phosphorus tribromide is added in portions to $52 \mathrm{~g}$. of phenoxybutyl alcohol. The mixture is cooled in ice during the reaction. After all of the phosphorus tribromide has been added, the mixture is allowed to stand a few hours and is then warmed on the steam-bath. The reaction mixture is poured into water and the phenoxybutyl bromide is taken up in a little benzene. The solution is dried over a little calcium chloride and distilled under reduced pressure. The yield is $50 \mathrm{~g} .(70 \%)$ of a product boiling at $153-$ $156^{\circ}$ at $18 \mathrm{~mm}$.

The Action of Hydriodic Acid on Phenoxybutyl Alcohol.-A mixture of $38 \mathrm{~g}$. of phenoxybutyl alcohol and $120 \mathrm{cc}$. of constant-boiling hydriodic acid (sp. gr. 1.70) is refluxed vigorously for 12 hours. The product is then worked up as described under the treatment with hydrobromic acid. From the reaction mixture $18.5 \mathrm{~g} .(26 \%)$ of tetramethylene iodide boiling at $147-152^{\circ}$ at $26 \mathrm{~mm}$. and $43 \mathrm{~g} .(67 \%)$ of phenoxybutyl iodide boiling at $165-172^{\circ}$ at $19 \mathrm{~mm}$. are obtained. The latter compound solidified to a product melting at $42-42.5^{\circ}$. The literature gives the melting point as $43-44^{\circ} .11$

The Action of Hydriodic Acid on Phenoxybutyl Iodide ${ }^{12}$.-By refluxing $43 \mathrm{~g}$. of phenoxybutyl iodide with $60 \mathrm{cc}$. of hydriodic acid (sp. gr. 1.70) for 7 hours and working up the reaction mixture in the usual way there is obtained $34 \mathrm{~g} .(70 \%)$ of tetramethylene iodide. Twelve $\mathrm{g}$. of phenoxybutyl iodide is recovered.

\section{Summary}

1. An improved method of preparation for the 1,4-dihalogen derivatives of butane has been developed which makes these substances more readily available for research work.

2. The method of Levene and Allen for reducing esters to alcohols has been somewhat improved so that yields of $62-75 \%$ of the theoretical amount of alcohol can be obtained from the higher esters.

URBANA, ILLINOIS

11 Ber., 39, 4361 (1906).

12 Ref. 11, p. 4362. 